\title{
Predicting Metamorphic Changes In Parkinson's Disease Patients Using Machine Learning Algorithms
}

\author{
G.Prema Arokia Mary', N.Suganthi', M.S.Hema ${ }^{3}$, M.Hari \\ Dharshini ${ }^{4}$, K.Vaishaali ${ }^{5}$, M.Monika Sri ${ }^{6}$ \\ ${ }^{1}$ Assistant Professor, Department of Information Technology, Kumaraguru \\ College of Technology, Coimbatore, India. \\ ${ }^{2}$ Professor, Department of Computer Science and Engineering, Kumaraguru \\ College of Technology, Coimbatore, India. \\ ${ }^{3}$ Associate Professor, Department of Information Technology, Anurag University, \\ Hyderabad, India. \\ ${ }^{4,5,6}$ Student, Department of Information Technology, Kumaraguru College of \\ Technology, Coimbatore, India.
}

\section{ABSTRACT}

Parkinson's disease is a nervous disorder mainly it affects the motor activities of the human body. Manifestations start step by step; at later point it becomes the greatest obstacle to do our day to today activities. Individuals influenced with Parkinson's ailment should go through lifestyle changes and enthusiastic changes like dozing issues, disposition swings, stultification, and skin issues. The proposed methodology is to analyse the proportion of metamorphic changes of a person affected by Parkinson's disease using machine learning techniques. Principal Component Analysis (PCA), recurrent neural network and logistic regression algorithms are used for prediction. The accuracy, precision, recall and F1 measure is used to assess the performance of the prediction algorithms. The dataset includes activities of daily living which from PPMI (Parkinson's Progression Markers Initiative) was taken for experimentation. Logistic regression can predict metamorphic changes with a higher accuracy of 92\% for sleep dataset and 95\% for 0lfactory(smell) dataset when compared to other two algorithms.

KEY WORDS: HALLUCINATIONS, LOGISTIC REGRESSION, METAMORPHIC CHANGES, NORMALIZATION, PCA, PREDICTION, RNN, TRANSFORMATION.

\section{INTRODUCTION}

Parkinson's Disease (PD) is a progressive nervous system disorder which affects the movement of the human beings. PD occurs due to lack of dopamine chemical

\section{ARTICLE INFORMATION}

*Corresponding Author: premaarokiamary.g.it@kct.ac.in Received 5th Oct 2020 Accepted after revision 26th Nov 2020 Print ISSN: 0974-6455 Online ISSN: 2321-4007 CODEN: BBRCBA

Thomson Reuters ISI Web of Science Clarivate Analytics USA and Crossref Indexed Journal

\section{Clarivate
Analytics}

NAAS Journal Score 2020 (4.31) SJIF: 2020 (7.728)

A Society of Science and Nature Publication,

Bhopal India 2020. All rights reserved.

Online Contents Available at: http//www.bbrc.in/

Doi: $h t t p: / / d x$.doi.org/10.21786/bbrc/13.11/30 in the human brain (Sontheimer H, 2015). Dopamine is a contraction of 3,4 dihydroxy phenethylamine. It comprises about $80 \%$ of the catecholamine content. It is also synthesized in plants and animals. It functions as a neurotransmitter in the brain (G.Prema Arokia Mary et al.., 2020). It is a chemical which sends signals to nerve cells and is released by neurons. Association network of protein builds the hereditary network collaboration of PD. There is no separate test for PD prediction. The PD is predicted based on the symptom's tests and clinical trials. Symptoms starts gradually in earlier stages. Early stages may be mild and unnoticed. Parkinson's disease signs and symptoms are different for everyone. Tremors 
are the most common one, but it also causes stiffness, slowing of movement and voice changes.

People usually develop this disease at the age of 60 or above. But now days young adults also affected with PD due to various factors such as environmental triggers, exposure to toxins like pesticides, smoking, head injury etc.,. Genetics also play a major role in causing PD. Young Onset Parkinson's disease (YOPD) occurs in people younger than 50 years. Youngsters also have symptoms similar to aged people like tremors in hands, arms, legs, jaw and face, rigid muscles, bradykinesia, impaired postures and balance, loss of automatic movements, speech changes etc., (Suwijn, Sven R. et al.., 2020). People with YOPD may experience non-motor symptoms including depression, sleep disturbances, anxiety, difficulty in swallowing, distorted sense of smell, unintentional writing, weight loss, stultification etc., (Feigl, Beatrix et al.., 2020, Tremblay, Cécilia et al.., 2020). The PD stages are divided into five different stages.

Symptoms of earlier stage or stage one is tremor, experiencing changes in walking and facial expressions. In stage two, people with PD experience rigidity and other movement symptoms on both sides of the body. In third stage, people face with postural disability which means movements becomes very slow, but one can manage by their own without any assistance. In stage four, people can severely affect by movement disorder. The person cannot be able to do their daily tasks, so they require a nursing care for all the activities. The fifth stage is said to be an advanced stage person become bedridden. People may experience hallucinations and delusions (Huang, Xuemei et al.., 2020).

Melancholy happens in roughly $40 \%$ of patients with Parkinson's illness. Sleep disturbances are the foremost characteristics of the non-motor issues of Parkinson's disease (PD) and the prevalence with advancing disease become greater. There are various causes of sleep disturbance in PD, and it has several factors that contributes to many patients. These can be widely classified into those which includes nocturnal sleep and daytime illustrations. The primary manifestation of PD is excessive daytime sleepiness which reflects the constructed areas which affected by the neurodegenerative process. The mental concomitant in Parkinson's disease is anxiety disturbances which confers to significant impairments in cognitive, functional, motor areas (Cummings, J. L., 1992).

It results in decreased quality of life, greater levels of care dependency and rises caregiver burden. Many of the people with PD would experience generalized anxiety disorder, anxiety attacks and social anxiety disorders. Most people who have PD, experienced a difficulty in swallowing which is called dysphagia, it can happen at any stage of Parkinson Disease (DeMaagd G, Philip A, 2015). Symptoms which include in swallowing difficulties are coughing or throat clearing during eating and drinking and difficulty while swallowing certain foods or liquids. Aspiration pneumonia is the cause of death in PD. People who experience the ability to smell which was called as dysosmia. It is an early sign of Parkinson (Laperle, A.H., Sances, S., Yucer, N, 2020). Modelling tools are used to study the disease and give prognostication of symptoms. To understand and identify the symptoms of the disease, quantitative studies and models suggested by some authors. The amalgamation of Parkinson's different data from several sources which are multiplex in nature and provide opportunities to study the premature stages of the patients, track the development and offering solutions. Heterogeneous data are handled using Big data analytics (S. Kanagaraj et al.., 2019).

The main contribution of the proposed methodology is to predict the metamorphic changes of the PD patients. The performance of the prediction algorithms is compared. This paper has been proceeded in such a way : apart from introduction along with literature section, second section consists of the proposed methodology, dataset information, data pre-processing and the implementation of three algorithms like PCA, RNN and Logistic Regression. Results are discussed in section three. The conclusion is presented in section four.

\section{MATERIAL AND METHODS}

Proposed Methodology: The proposed methodology for PD prediction using machine learning algorithms. Three algorithms are used for prediction namely Recurrent Neural Networks, Logistic Regression and Principal Component Analysis. The steps involved in the proposed methodology are importing libraries, loading the Data, data pre-processing, splitting the data as train data and test data, prediction algorithm implementation and performance assessment such as accuracy, precision, recall and f1_score calculation. Two datasets i.e. sleep disorder and olfactory (smell) datasets is taken for implementation.

Dataset Information: The dataset is taken from PPMI (Parkinson's Progression Markers Initiative). PPMI is a milestone observational clinical investigation to exhaustively assess partners of critical enthusiasm utilizing progressed imaging, biologic inspecting and clinical and social evaluations to recognize biomarkers of Parkinson's disease movement. The informational collection for dozing problem and olfactory smell is taken. The informational index contains 8367 information for Parkinson malady influenced individuals.

Data Pre-processing: Data pre-processing is an important step in machine learning that helps to strengthen the aspects of data to extract the meaningful insights from the data. It is a technique of converting the raw data to quality data. Normally raw data is incomplete, inconsistent, or it has some error or outliers. The steps involved in data pre-processing is: select the dataset, import all the crucial libraries, Import the dataset, data cleaning, data reduction and splitting. In data cleaning, the missing values, null values are identified and 
removed. The data inconsistencies also removed in data cleaning. The data set has a greater number of features. All the features are not required for prediction. The irrelevant features may degrade the performance of the prediction algorithm. The required feature selection is done in data reduction. Next comes data splitting, here the data is divided into two sets namely training set and test set. The training set is used to build the model. The test set is used to check performance of the model (K. Saranya et al.., 2014).

\section{Algorithm Introduction}

Principal Component Analysis: Principal component analysis (PCA) is a technique in which data with high dimensional are lowered to low dimension by selecting the most important features that capture maximum information about the dataset (S. Sehgal et al.., 2014). Often People think that PCA takes only the selected features and discards the remaining features. But it actually builds the new set of properties based on the older ones. PCA act as powerful tool in analysing the data. The main advantage of this algorithm reduces with a smaller number of features (Rao, C. Radhakrishna, 2020). First normalization process is done so that a dataset is normalized before applying PCA algorithm. Covariance Matrix (CM) is calculated as:

$\mathrm{CM}=[\mathrm{Va}[\mathrm{Z} 1] \quad \mathrm{Cv}[\mathrm{Z} 1, \mathrm{Z} 2] \quad \mathrm{Cv}[\mathrm{Z} 2, \mathrm{Z} 1] \quad \mathrm{Va}[\mathrm{Z} 2] \quad]$

$\mathrm{Va}[\mathrm{Z} 1]=\mathrm{Cv}[\mathrm{Z} 1, \mathrm{Z} 2]$ and $\mathrm{Va}[\mathrm{Z} 2]$

Eigenvalues and eigenvectors are as calculated to generate CM, $\lambda$ is the eigenvalue of matrix $H$

$\mathrm{D}(\lambda \mathrm{I} 1-\mathrm{H})=0$

where,

I1 denotes dimensions of identity matrix are equal

D denotes determinant of a matrix

For every eigenvalue $\lambda$, a relating eigenvector v1,

$(\lambda \mathrm{I} 1-\mathrm{H}) \mathrm{v} 1=0$

The components are chosen, and Feature vector (FV) is formed as,

$\mathrm{FV}=($ eig1,eig2)

where,

eig1, eig2 are eigenvalues

Principal Components can be formed as follows:

NewData $=\mathrm{F}^{\mathrm{VT}} \times$ ScaledData $^{\mathrm{T}}$

(6) $\mathrm{K}_{\mathrm{a}-1}$ denotes current state

where,

New Data is a matrix which consists principal components
$\mathrm{L}_{\mathrm{a}}$ denotes input state $\mathrm{n}$

Scaled Data is an original dataset of scaled version

$\mathrm{T}$ is transpose of matrix

After implementing PCA algorithm, the performance measures like accuracy, recall, precision, F1 score are to be calculated.

Recurrent Neural Network: A Recurrent Neural Network (RNN) is a type of artificial neural networks where the interconnections from one node to another node constructs a directed graph. It is derived from feedforward neural network policy. RNN algorithm gives predictive results in sequential data. RNN algorithm used in developing the models which does similar activity of neurons in the human brain. Some applications of RNN are Robot control, Speech recognition, Grammar learning etc., also used by Google's voice search. It perfectly suits for machine learning problems and its the first algorithm which recollects the input. For the past few years, it is one of the best algorithms which has seen excellent results in deep learning. It consists of cyclic connections which make the algorithm a more efficient and powerful tool to model the sequential data. It achieved a great success while demonstrating prediction tasks like handwriting recognition and language modelling. It is performed and obtained great results in sequence labelling (Z. Tang, et al.., 2016).

First of all, independent activations are converted into dependent activations by giving similar loads to each layer. By this conversion intricacy of parameters can be decreased. The last output is always remembered, because previous outputs are given as input to the next hidden layer. Likewise, all layers are joined together, and weights remains same for every layer and it is converted into a single recurrent layer (Salehinejad, Hojjat et al.., 2017). By feeding the initial data to the network, current state can be calculated with the help of multiple outputs which taken from the previous state. It can have many steps according to the problem and once everything is done, the output is calculated which is done in final state. Then comparison between output and actual output (target output) takes place and weight gets updated. Now RNN is trained. At time $t$, it produces output and the parameter affected was available at time $t+1$. It has a remarkable representation so that it keeps the information about the past steps. In such a way, it keeps the recent past and present inputs to produce the output for the data. The current state is calculated by the formula:

$\mathrm{K}_{\alpha}=\mathrm{f}\left(\mathrm{K}\left({ }_{\mathrm{a}-1}\right), \mathrm{L}_{\mathrm{a}}\right)$

Activation function is applied by the formula (tanh):

$\left.\mathrm{K}_{\alpha}=\tanh \left(\mathrm{Y}_{\mathrm{kk}} \mathrm{K}_{(\mathrm{a}-1}\right)+\mathrm{Y}_{\mathrm{lk}} \mathrm{L}_{\mathrm{a}}\right)$ 
whereas,

$\mathrm{Y}_{\mathrm{lk}}$ denotes weight at input neuron

The result $\mathrm{Zt}$ is calculated by using the formula:

$\mathrm{Z}_{\mathrm{t}}=\mathrm{Y}_{\mathrm{kz}} \mathrm{K}_{\mathrm{a}}$

whereas,

Ykz denotes weight at output layer

Logistic Regression Algorithm: Logistic regression is one of the famous algorithms to forecast dichotomous values. It predicts the probability of the outcomes (Peng, Joanne et al.., 2002). The nature of target variable is dichotomous, which means that there would be only two possible classes. In general, the target variable is in binary form and the desired outcome is either 0 or 1 . Logistic Regression is one of the simplest ML algorithms
$\mathrm{Y}_{\mathrm{kk}}$ denotes weight at recurrent neuron

that can be used for various classification problems (X. Zou et al.., 2019). The general equation to calculate linear regression is:

$\mathrm{s}(\mathrm{Ex}(\mathrm{M}))=\mathrm{A}+\mathrm{Bi} 1+\mathrm{Ci} 2$

where,

$\mathrm{s}()$ is link function

$\operatorname{Ex}(\mathrm{M})$ is expectation of target variable.

$\mathrm{A}+\mathrm{B}^{*} \mathrm{i} 1+\mathrm{C}^{*} \mathrm{i} 2$ is linear predictor.

From this logistic regression equation can be derived and the equation is:

$$
\begin{aligned}
\log \log \left(\frac{p}{1-p}\right) & \\
& =B_{0} \\
& +B(\text { Age })
\end{aligned}
$$

Table 1. Performance comparison chart of Accuracy, Recall, Precision, F1_Score values for PCA, Logistic regression \& RNN algorithm

\begin{tabular}{|l|c|c|c|c|c|c|}
\hline \multirow{2}{*}{$\begin{array}{l}\text { Performance } \\
\text { Evaluation }\end{array}$} & \multicolumn{3}{|c|}{ PCA Algorithm } & \multicolumn{2}{|c|}{$\begin{array}{c}\text { Logistic Regression } \\
\text { Algorithm }\end{array}$} & \multicolumn{2}{l|}{ RNN Algorithm } \\
\cline { 2 - 7 } & $\begin{array}{c}\text { Sleep } \\
\text { Dataset }\end{array}$ & $\begin{array}{c}\text { Smell } \\
\text { Dataset }\end{array}$ & $\begin{array}{c}\text { Sleep } \\
\text { Dataset }\end{array}$ & $\begin{array}{c}\text { Smell } \\
\text { Dataset }\end{array}$ & $\begin{array}{c}\text { Sleep } \\
\text { Dataset }\end{array}$ & $\begin{array}{c}\text { Smell } \\
\text { Dataset }\end{array}$ \\
\hline Accuracy & $83 \%$ & $80 \%$ & $92 \%$ & $95 \%$ & $89 \%$ & $86 \%$ \\
\hline Recall & $78 \%$ & $76 \%$ & $90 \%$ & $95 \%$ & $87 \%$ & $85 \%$ \\
\hline Precision & $80 \%$ & $76 \%$ & $87 \%$ & $90 \%$ & $85 \%$ & $86 \%$ \\
\hline F1 Score & $79 \%$ & $74 \%$ & $85 \%$ & $91 \%$ & $84 \%$ & $82 \%$ \\
\hline
\end{tabular}

After training phase, test dataset prediction takes place. Once the prediction phase successfully completed the performance measures like accuracy, confusion matrix, precision, F1 score are calculated.

\section{RESULTS AND DISCUSSION}

Sleep and smell dataset are taken from PPMI repository. The attributes chosen to predict sleep disorder are Vivid dreams, Aggressive Dreams, Movement awaken and sleep disturbances and for Olfactory(smell) the attributes of scent levels from 1 to 5 and the total percentage are considered. Total 8367 instances taken for implementation. The language used for implementation is Python. In data pre-processing all the null values and missing records are removed. All the attributes are taken for prediction. Principal Component Analysis, Logistic Regression and in Recurrent Neural Network prediction algorithms are implemented. The accuracy, precision, F1 score and recall for sleep and smell dataset in each algorithm is given in table 1 .

$\operatorname{Accuracy}(\mathrm{A})$ is determined by using the formula,

$A=\frac{Q+P}{Q+P+R+S}$

Recall (R) is determined by using the formula,

$$
R=\frac{P}{P+S}
$$

F1 score $(\mathrm{F})$ is computed as,

$$
F=2 *\left(\frac{P * R}{P+R}\right)
$$

Whereas,

$\mathrm{P}$ is said to be true positive

$\mathrm{Q}$ is said to be true negative

$\mathrm{R}$ is said to be false positive

$\mathrm{S}$ is said to be false negative

The logistic regression gives better performance in both sleep dataset and smell dataset when compared to other two algorithms which is shown in Figure 2.

Precision(P) is determined by using the formula, 
Figure 1: Graph depicts the Sleep and Smell data by Performance Evaluation of PCA,Sleep data(skyblue colour),Smell data(darkblue colour) where, $\mathrm{X}$-axis shows the accuracy,Precision,F1-Score, Recall and Y-axis shows the percentage values.

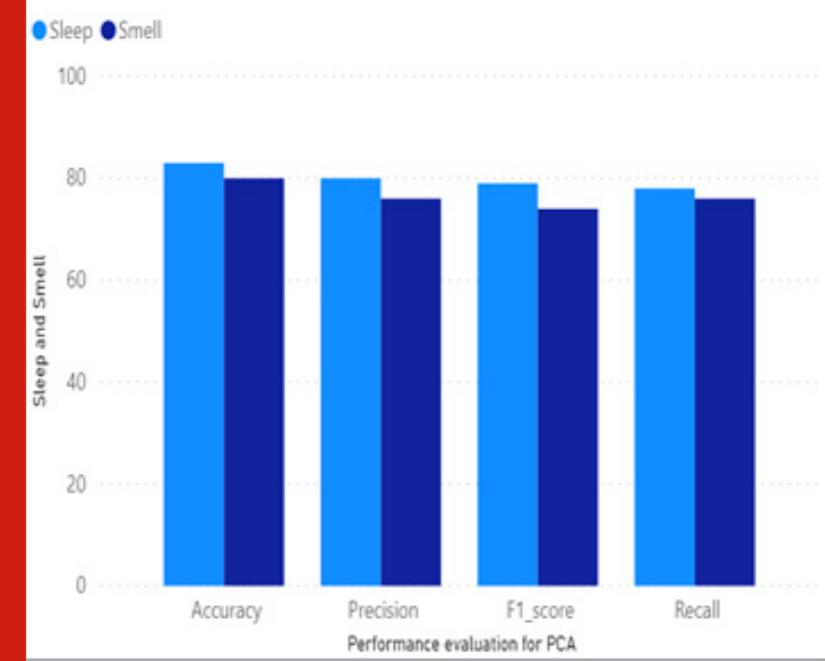

Figure 3: Graph depicts the Sleep and Smell data by Performance Evaluation of RNN Sleep data(maroon colour),Smell data(purple colour) where, $\mathrm{X}$-axis shows the accuracy,Precision,F1-Score, Recall and Y-axis shows the percentage values.

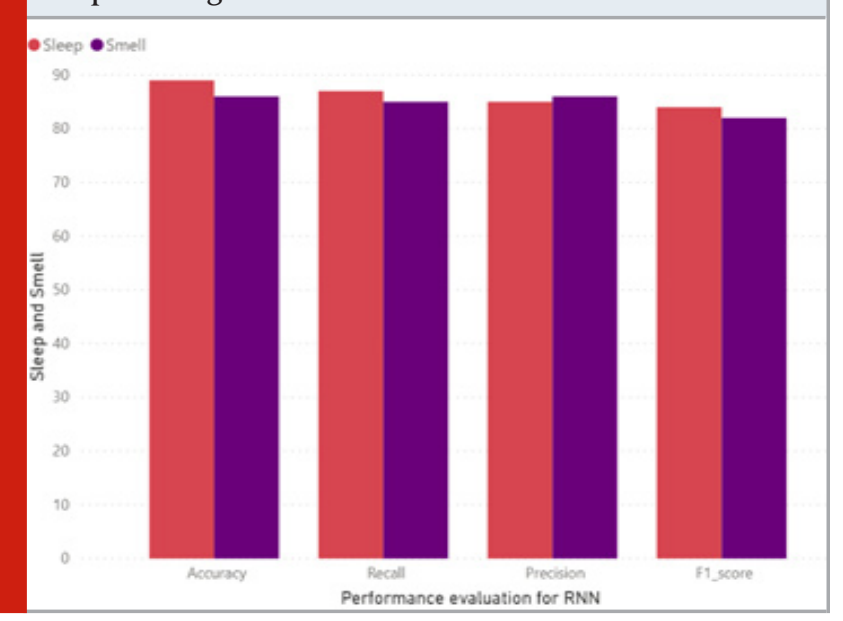

\section{CONCLUSION}

The prediction of PD using machine leaning techniques is proposed and implemented. Three algorithms such as principal component analysis, recurrent neural network and logistic regression algorithms are implemented. The performance of these three algorithms are compared. The logistic regression algorithm has highest accuracy of 92\% for sleep dataset and 95\% for smell dataset.

\section{REFERENCES}

Cummings J. L. (1992). 'Depression and Parkinson's disease: a review'. The American journal of
Figure 2: Graph depicts the Sleep and Smell data by Performance Evaluation of Logistic Regression Sleep data(orange colour),Smell data(yellow colour) where, $\mathrm{X}$-axis shows the accuracy,Precision,F1-Score, Recall and Y-axis shows the percentage values.

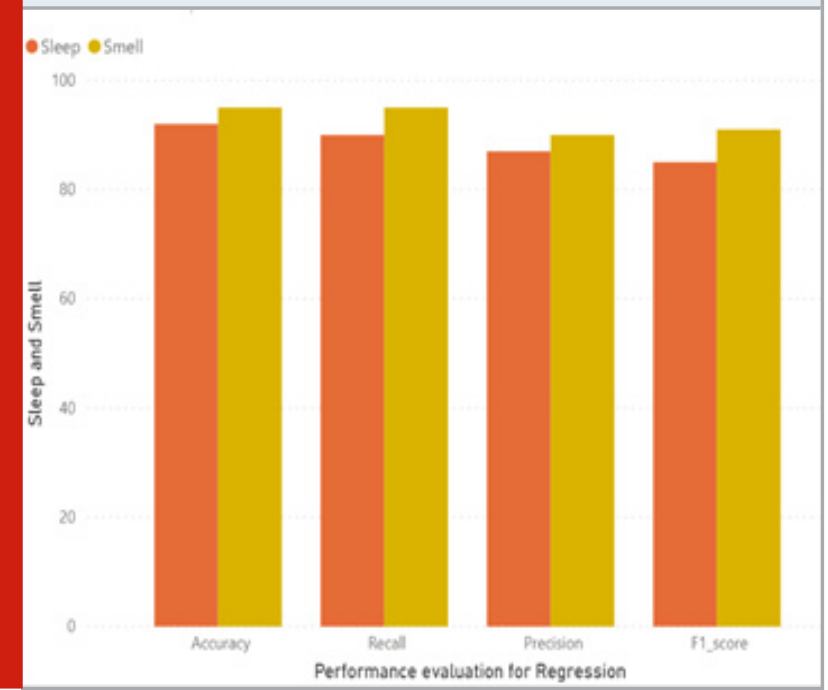

psychiatry, 149(4), 443-454. https://doi.org/10.1176/ ajp.149.4.443

DeMaagd, G., \&t Philip, A. (2015). 'Parkinson's Disease and Its Management: Part 1: Disease Entity, Risk Factors, Pathophysiology, Clinical Presentation, and Diagnosis'. P \& T : a peer-reviewed journal for formulary management, 40(8), 504-532.

Feigl B, Dumpala S, Kerr GK, Zele AJ (2020) 'Melanopsin Cell Dysfunction is Involved in Sleep Disruption in Parkinson's Disease'. J Parkinsons Dis ,10(4) 1467-1476. doi:10.3233/jpd-202178. PMID: 32986681.

Huang, Xuemei et al., (2020), 'The D 1/D 5 Dopamine Partial Agonist PF-06412562 in Advanced-Stage Parkinson's Disease: A Feasibility Study', J Parkinsons Dis, 10(4) 1515-1527. doi:10.3233/jpd-202188. PMID: $32986682.1-13$

Kanagaraj. S, Hema. M.S, Nageswara Gupta. M. (2019) 'Machine Learning Techniques for Prediction of Parkinson's Disease using Big Data', Volume-8 Issue10, International Journal of Innovative Technology and Exploring Engineering (IJITEE).

Laperle, A.H., Sances, S., Yucer, N. et al., (2020) 'iPSC modelling of young-onset Parkinson's disease reveals a molecular signature of disease and novel therapeutic candidates',Nature Medicine,26(2), 289-299, https://doi. org/10.1038/s41591-019-0739-1.

Peng, Joanne \& Lee, Kuk \&t Ingersoll, Gary. (2002) 'An Introduction to Logistic Regression Analysis and Reporting,, Journal of Educational Research - J EDUC RES. 96. 3-14. 10.1080/00220670209598786.

Prema Arokia Mary. G, Saru Priya. K, Suganthi. N, 
Sathyavathi. S. (2020), 'Dopamine Analysis Using Multiple Machine Learning Techniques', International Journal of Advanced Science and Technology, 29(05), 10220 - 10227.

Rao, Radhakrishna .C. (2020) 'The Use and Interpretation of Principal Component Analysis in Applied Research', The Indian Journal of Statistics, JSTOR, Sankhy囚. vol. 26, no. 4, 1964, pp. 329-358.

Salehinejad, H., Baarbe, J., Sankar, S., Barfett, J., Colak, E., \&t Valaee, S. (2018). 'Recent Advances in Recurrent Neural Networks'. ArXiv, abs/1801.01078.

Saranya, K. \&t Hema, M.S. \&t Chandramathi, S.. (2015). 'Data fusion in ontology based data integration. 2014 International Conference on Information Communication and Embedded Systems', ICICES 2014. 10.1109/ICICES.2014.7033792.

Sehgal, S., Singh, H., Agarwal, M., Bhasker, V., \&t Shantanu (2014). Data analysis using principal component analysis. 2014 International Conference on Medical Imaging, m-Health and Emerging Communication Systems (MedCom), 45-48.

Sontheimer. H (2015) Parkinson Disease. Diseases of the Nervous System: Academic Press 133-64.
Suwijn, S. R., Samim, H., Eggers, C., Espay, A. J., Fox, S., Lang, A. E., Samuel, M., Silverdale, M., Verschuur, C., Dijk, J. M., Verberne, H. J., Booij, J., \&t de Bie, R. (2020) 'Value of Clinical Signs in Identifying Patients with Scans without Evidence of Dopaminergic Deficit (SWEDD)', Journal of Parkinson's disease, 10(4), 15611569. https://doi.org/10.3233/JPD-202090.

Tang. Z, Wang. D \& Zhang. Z. (2016)'Recurrent neural network training with dark knowledge transfer', IEEE International Conference on Acoustics, Speech and Signal Processing (ICASSP), Shanghai.

Tremblay, C., Iravani, B., Aubry Lafontaine, É., Steffener, J., Fischmeister, F., Lundström, J. N., \&t Frasnelli, J. (2020). Parkinson's Disease Affects Functional Connectivity within the Olfactory-Trigeminal Network. Journal of Parkinson's disease, 10(4), 1587-1600. https://doi.org/10.3233/JPD-202062.

Zou. X, Hu. Y, Tian .Z \& Shen. K. (2019) 'Logistic Regression Model Optimization and Case Analysis',IEEE 7th International Conference on Computer Science and Network Technology (ICCSNT), Dalian, China, pp. 135139, doi:10.1109/ICCSNT47585.2019.8962457. 